Revista de Negócios_ISSN 1980.4431_vol. 18, n.3, p.3_20, 2013_DOI:10.7867/1980-

431.2013v18n3p3_20

\title{
A implementação de um departamento de pós-vendas em um provedor de serviços de saúde e o estreitamento dos relacionamentos com os clientes
}

\section{The implementation of an after sales department in a health care service provider and the relationships tightening with customers}

Gabriel Sperandio Milan

UCS Universidade de Caxias do Sul - Brasil

gabmilan@terra.com.br

Recebido em 14 de junho de 2012. Alteração em 4 de fevereiro de 2013. Aprovado em 25 de fevereiro de 2013

Editor Responsável: Edson Roberto Scharf, Dr.

Processo de avaliação por double blind review

\section{Resumo}

O presente trabalho é resultado de uma pesquisaação desenvolvida em uma operadora de planos de saúde com atuação local, apresentando as etapas e os principais resultados provenientes da implementação de um projeto de estruturação de um Departamento de Pós-vendas para o Plano de Saúde em estudo. O objetivo central do projeto é o estabelecimento de contatos sistemáticos com as empresas, uma vez que o foco está nos clientes do Plano Empresarial, fortalecendo relacionamentos de longo prazo e maximizando a satisfação e a retenção de clientes. Neste sentido, rompe-se com o paradigma tradicional de que as atividades pósvendas estejam somente direcionadas ao tratamento de reclamações. Embora o tratamento de reclamações seja imprescindível para a manutenção e/ou melhoria nos níveis da qualidade dos serviços prestados e da satisfação de clientes, tal atividade deve ter como principal fundamento uma filosofia baseada no marketing de relacionamento, aumentando a proximidade com os clientes.

Palavras-chave: pós-vendas, qualidade em serviços, marketing de relacionamento, satisfação de clientes, retenção de clientes.

\begin{abstract}
The present work is resulted of an action research developed in a health care plan with local performance, presenting the stages and main results from the implementation of a structured project of an After Sales Department for the Health Care Plan studied. The central aim of the project is the establishment of systematic contacts with the companies, so that the focus is in the customers of the Enterprise Plan, strengthening long-term relationships and maximizing the customer satisfaction and the customer retention. In this way, it is breached with the traditional paradigm of that the activities after sales only are directed to the treatment of claims. Although the treatment of claims is essential for the maintenance and/or improvement in the quality levels of services rendering and customers satisfaction, such activity must have as main bedding a philosophy based on the relationship marketing, increasing the proximity with customers.
\end{abstract}

Keywords: after sales; service quality; relationship marketing; customer satisfaction and retention. 
A implementação de um departamento de pós-vendas em um provedor de serviços de saúde e o estreitamento dos relacionamentos com os clientes

\section{Introdução}

Diversos fatores vêm pressionando as empresas brasileiras que atuam no setor da saúde a qualificar seus processos de gestão. Dentre eles, emerge o aumento da demanda, demanda esta que o governo, por falta de estrutura, repassa a responsabilidade para a iniciativa privada, expandindo as coberturas obrigatórias por meio da nova regulamentação, de acordo com a Lei 9.656/98, publicada no Diário Oficial em 04 de junho de 1998.

Além disso, outros fatores que pressionam o setor da saúde são: a queda nas taxas de fertilidade e de mortalidade infantil, em contrapartida a uma maior longevidade dos idosos, o ressurgimento de moléstias erradicáveis, o aparecimento de novas doenças contagiosas e outras relacionadas aos hábitos de vida ou doenças como câncer, cardiopatias, depressão, obesidade e doenças emocionais, índices crescentes quanto à poluição, maior pressão quanto à qualificação dos profissionais ligados à saúde e ao emprego de novas tecnologias, sem falar no impacto das variáveis econômicas e no aumento dos custos advindos de pesquisa e implementação de novos medicamentos, protocolos de atendimento e equipamentos; obrigando as empresas a maximizar suas estruturas e parcerias, em busca de uma maior competitividade no mercado.

Como na área da saúde os custos vêm sendo os direcionadores primários das mudanças, acirrando a competição por clientes e seus recursos, reforça-se a necessidade de reduzir ou eliminar custos desnecessários e de preservar e incrementar a qualidade dos serviços, resultando em satisfação para os clientes, assegurando, desta forma, a viabilidade econômica das organizações (CLINEFELTER, 2000; BISOGNANO, 2000; FOLLAND; GOODMAN; STANO, 2008; KOTLER; SHALOWITZ; STEVENS, 2010). É oportuno comentar que, no Brasil, estimase que uma quarta parte da população está associada ou conveniada a algum tipo de plano de saúde privado (BAHIA; LUIZ, 2006).

Por outro lado, é imprescindível entender que o comportamento do cliente mudou. Ao possuir acesso a uma vasta gama de informações o cliente exerce, cada vez mais, sua liberdade ao escolher seu provedor de saúde (WOLF, 2001a). Assim, uma das estratégias que organizações do setor da saúde podem implementar para atingir seus objetivos, e até mesmo construir a retenção e a lealdade dos clientes, é cultivar relacionamentos duradouros (WOLF, 2001a; 2003; KOTLER; SHALOWITZ; STEVENS, 2010; CHAHAL; KUMARI, 2011; GAUR et al., 2011). Por isso, um atendimento diferenciado e focado na retenção de clientes (COELHO; HENSELER, 2012; LIN; WU, 2011) faz com que a busca pelo estreitamento e consolidação dos relacionamentos existentes entre uma empresa e seus clientes a partir da agregação de valor entre as partes com base em serviços pósvendas seja vital para o sucesso das empresas (MILAN; RIBEIRO, 2003; MILAN, 2006; VAVRA; PRUDEN, 1995).

É importante ressaltar que os serviços de saúde são atividades baseadas em relacionamentos (WOLF, 2001a; 2001b; MALLOCK; LUSSIER, 2000; CHAHAL; KUMARI, 2011) e, nesta direção, as organizações devem reconhecer que a satisfação e, em especial, a retenção e, se possível, a lealdade de clientes pode gerar um valor significativo em termos de retorno sobre o investimento ao longo do tempo (HESKETT et al., 1994; REICHHELD, 1996a; 1996b; REICHHELD; SASSER Jr., 1990; REICHHELD; MARKEY Jr.; HOPTON, 2000; LAM et al., 2004; MILAN; TREZ, 2005; MILAN, 2006; PIVA et al., 2007; EDWARD; SHADEV, 2011; WILLIAMS; NAUMANN, 2011; MILAN; DE TONI, 2012, dentre outros).

Considerando tal contexto, ao se desenvolver uma pesquisa-ação, o objetivo central do estudo foi o de detalhar a forma 
com que uma empresa da área da saúde implantou o seu departamento de pósvendas, contemplando, inclusive, a avaliação dos resultados obtidos após dois anos de atuação da equipe. Para tanto, foram coletadas evidências, como, por exemplo, dados coletados de relatórios disponibilizados pela área e algumas percepções de clientes, que apontam que tal iniciativa repercutiu em uma maior proximidade relacional entre as partes e na diminuição no volume de reclamações e na perda de clientes.

\section{A relação entre pós-vendas e a reten- ção de clientes por meio da prática do marketing de relacionamento}

Para Vavra e Pruden (1995), pósvenda é uma filosofia que enfatiza o estabelecimento de relacionamentos de valor com clientes, salientando que as atividades pertinentes ao pós-vendas são uma prática cujo foco é a retenção e, se possível, a construção da lealdade dos clientes (OLIVER, 2010; KERNS; ST. CLAIR, 2000; MILAN; RIBEIRO, 2003; MILAN, 2006; WILSON, 2008), sendo que o grande desafio é reconhecê-los, mostrando a eles o quanto a empresa os estima por terem lhe conferido sua preferência.

Embora o nível de competição intensificado no setor da saúde tenha aumentado o foco na satisfação do cliente (EISENBERG, 1997; GREGÓRIO, 2009; SPILLER et al., 2009), a qual provém de sua avaliação sobre qualidade, valor pago e experiência do atendimento (CASANOVA, 1999; GAUR et al., 2011), tal mudança de postura empresarial deve ser aprofundada, consolidando um novo modo de fazer negócios, passando de uma visão de transação discreta, com ênfase na venda, para uma troca relacional, baseada em relacionamentos de longo prazo (DWYER, SCHURR; OH, 1987; MORGAN; HUNT, 1994; GUMMESSON, 1998; MILAN, 2004;
2006; MILAN; RIBEIRO, 2003; MILAN; TREZ, 2005; MILAN; DE TONI, 2012). Evidencia-se, portanto, que os serviços pós-vendas se caracterizam como uma prática relacional, a qual está fundamentada nos esteios do marketing de relacionamento (MILAN; RIBEIRO, 2003; MILAN, 2006).

Neste sentido, Vavra (1994) postula, com propriedade, que por quanto mais tempo se mantém um cliente, mais lucrativo o cliente é para a empresa, e por quanto mais tempo o cliente repetir negócios com uma mesma organização, mais dependente se tornará de seus produtos e serviços e menos suscetível ficará a ofertas com preços mais baixos por parte da concorrência. Por isso, estimular e consolidar a implementação de ações fundamentadas no marketing de relacionamento pode conferir às empresas um modo mais consistente de competir no mercado, aproximando-se dos clientes. Neste contexto, o marketing de relacionamento pode ser entendido como um conceito filosófico-estratégico que pressupõe que as empresas devam atrair, manter e ampliar os relacionamentos existentes com os clientes (BERRY, 2002) no sentido de se tornarem mais competitivas perante a concorrência.

Consoante isso, Grönroos (2000) afirma que o marketing de relacionamento é um processo de identificar e estabelecer, manter, aumentar e, se necessário, terminar ou finalizar relacionamentos com clientes e outros stakeholders, com lucro, para que os objetivos de todas as partes envolvidas sejam alcançados, onde isto é feito por uma doação mútua e pelo cumprimento das promessas. Dessa forma, os relacionamentos com os clientes deveriam ser encarados como a "razão de ser" das organizações, e a filosofia e a prática do marketing deveriam estar direcionadas à construção e à ampliação de tais relacionamentos, com foco na retenção ou, até mesmo, na lealdade dos clientes, sob uma perspectiva de longo prazo. 
A implementação de um departamento de pós-vendas em um provedor de serviços de saúde e o estreitamento dos relacionamentos com os clientes

Para que a manutenção e o desenvolvimento desses relacionamentos seja algo possível, é imprescindível compreender o marketing de relaciona-mento, também, como um processo contínuo de envolvimento entre os parceiros de negócios em atividades cooperativas e colaborativas com o objetivo de criar ou aumentar o valor econômico mútuo a custo reduzido (PARVATIYAR; SHETH, 2000).

\section{O desenvolvimento da pesquisa e sua ambiência}

O presente trabalho foi desenvolvido sob a forma de uma pesquisa-ação, um tipo de pesquisa intervencionista na qual o pesquisador assume o papel de agente da mudança nos processos e eventos que está simultaneamente estudando (GUMMESSON, 2001), de acordo com os pressupostos encontrados na literatura (THIOLLENT, 2009; MEYER, 2000; MORRISON, 2001; HAIR Jr. et al., 2010; COOPER; SCHINDLER, 2011; SCOTT; GARNER, 2013).

No trabalho, foram implementadas a pesquisa documental (VERGARA, 2011; HENNINK; HUTTER; BAILEY, 2011), realizando análises e o aproveitamento de dados e informações provenientes de documentos e relatórios conservados e disponibilizados pela empresa (relatórios de cliente, registros de vistas a clientes e de atendimentos no departamento de pósvendas), juntamente com entrevistas individuais em profundidade, com uma abordagem semi-estruturada, mediante a aplicação de um Roteiro Básico de Questões (RIBEIRO; MILAN, 2004; KING; HORROCKS, 2010; HENNINK; HUTTER; BAILEY, 2011) com quatro gestores da empresa (Diretor Superintendente, Diretor Comercial, Gerente Comercial e Gerente Operacional), com uma duração média de 1 hora e 45 minutos.

O emprego destas técnicas de pesquisa permitiu um levantamento e avaliação da situação de pesquisa por meio da contextualização do nível de prestação de serviços da empresa em estudo e do grau de relacionamento que vinha praticando com os seus clientes, prestadores de serviço (terceiros) e colaboradores, aproveitando informações no que diz respeito a aspectos políticos, culturais e mercadológicos.

A fim de coletar dados e registrar a percepção de alguns clientes quanto aos resultados iniciais provenientes do projeto proposto, também foram realizadas entrevistas individuais em profundidade com três clientes, escolhidos aleatoriamente, em duas sessões cada, com duração média de 45 minutos para cada seção (Sessões 1 e 2), com uma abordagem semi-estruturada, mediante a aplicação de um Roteiro Básico de Questões. Cabe ressaltar que, no início da primeira sessão, foi realizada uma breve explanação dos objetivos do estudo e de como este seria desenvolvido, assegurando a cada participante (entrevistado) total sigilo e anonimato em relação às suas colocações. Posteriormente, foram resgatados excertos dessas entrevistas (subseção 5.5.2), trazendo a percepção dos clientes quanto à atuação da equipe de pós-vendas e seu impacto no relacionamento com o provedor de serviços.

Como ambiente para o estudo, utilizou-se uma operadora de planos de saúde com atuação local, focada no segmento Plano de Saúde Empresarial, e classificada na modalidade medicina de grupo. Em relação à sua estrutura, a empresa integra um Grupo constituído por cinco unidades de negócio: Plano de Saúde, Hospital, Medicina do Trabalho, Laboratório e Farmácias. Sua base de clientes é composta por 58.721 associados, sendo 42.145 provenientes do Plano Empresarial, distribuídos entre 1.527 empresas-cliente e representando $71,77 \%$ do total de associados, mais 16.576 do Plano Individual, representando 28,23\%. 


\section{Implementação do projeto de estrutu- ração do departamento de pós-vendas}

No intuito de obter os resultados pretendidos com a implementação do projeto de estruturação do Departamento de Pós-Vendas, observou-se algumas etapas, abordadas nas subseções que seguem:

\subsection{Definição do conceito utilizado na estruturação do Departamento de Pós- Vendas}

Como fundamentação conceitual para o projeto, partiu-se do entendimento de que as atividades pós-venda devem ser vistas como uma filosofia baseada no relacionamento com os clientes (VAVRA; PRUDEN, 1995; MILAN, RIBEIRO, 2003; WILSON, 2008). Assim sendo, o marketing de relacionamento emergiu como o principal arcabouço teórico na proposição do Departamento de PósVendas, uma vez que se baseia na estratégia de criação de valor para as partes envolvidas (GORDON, 2001; MILAN; RIBEIRO, 2003).

O conceito adotado foi $\mathrm{o}$ de estabelecer, manter e aprimorar relacionamentos com os clientes sob uma ótica de longo prazo (MORGAN; HUNT, 1994; VAVRA; PRUDEN, 1995; GUMMESSON, 1998; DAY, 2000; GRÖNROOS, 2000, KOTHANDARAMAN; WILSON, 2000; MILAN; RIBEIRO, 2003; MILAN, 2004; MILAN; DE TONI, 2012). Portanto, as atividades desempenhadas pela equipe de pós-vendas devem ser fundamentadas no marketing de relacionamento e objetivar a retenção de clientes.

\subsection{Objetivos e operacionalização do Departamento de Pós-Vendas}

Como objetivos propostos para o Departamento de Pós-Vendas, foram identificados:

a) estabelecer contatos com clientes no intuito de fortalecer um relacionamento de longo prazo e sua retenção;

b) consolidar a imagem institucional e a amplitude do mix de serviços;

c) detectar pontos fortes e fracos e oportunidades e ameaças em relação ao mercado;

d) auxiliar no agendamento e operacionalização das atividades do Grupo de Prevenção junto aos clientes;

e) encaminhar documentação relativa a reclamações para a gerência responsável e dar feedback ao cliente sobre as ações adotadas;

f) dirimir dúvidas para os clientes (empresas) e usuários (funcionários e/ou dependentes);

g) sensibilizar e estimular os clientes a solicitarem propostas de serviços que ainda não possuem;

h) levantar e registrar os motivos que causam a deserção de clientes; e

i) identificar sugestões para novos serviços.

Para que tais objetivos fossem alcançados, definiu-se que a operacionalização do Departamento de Pós-Vendas compreenderia um programa sistemático de visitas à carteira de clientes do Plano de Saúde Empresarial, estabelecendo um relacionamento estreito com as pessoas que exercem os papéis de decisor, negociador e influenciador no processo de permanência da empresa junto ao provedor de saúde.

\subsection{Ações necessárias para a imple- mentação do projeto}

Para a implementação do projeto, foi necessária a execução das seguintes ações ou atividades:

a) levantamento da carteira de clientes (segmento empresarial); 
A implementação de um departamento de pós-vendas em um provedor de serviços de saúde e o estreitamento dos relacionamentos com os clientes

b) estabelecimento de metas;

c) definição da estrutura de pessoal;

d) projeção do cronograma de implementação;

e) capacitação e definição das políticas de conduta da equipe;

f) planejamento e implantação do programa de visitas;

g) apoio da Direção e Gerências à equipe;

h) definição do processo para tratamento das reclamações e feedback aos clientes;

i) acompanhamento da solução de problemas e respostas aos clientes; j) controle e análise dos resultados;

k) avaliação da relevância das atividades pós-venda para a retenção de clientes; e

1) integração da equipe de pósvendas com as demais áreas da empresa.

\subsection{Metas de visitação, necessidade de pessoal e perfil dos colaboradores}

Ao projetar a meta de visitas, foi utilizada a análise da curva $\mathrm{A} / \mathrm{B} / \mathrm{C}$ de clientes, considerando $\mathrm{o}$ porte das empresas em relação ao número total de usuários, conforme consta na Tabela 1.

Tabela 1: Meta de visitação para o Departamento de Pós-Vendas

\begin{tabular}{crcc}
\hline Perfil dos Clientes & Empresas & Critério (visitas/ano) & Meta (visitas/ano) \\
\hline A - acima de 201 usuários & 23 & 4 & 92 \\
B - de 51 até 200 usuários & 90 & 3 & 270 \\
C - com até 50 usuários & 1.414 & 1 & 1.414 \\
TOTAL & 1.527 & - & 1.776 \\
\hline
\end{tabular}

Fonte: Relatório de Clientes - Plano de Saúde Empresarial.

Calculando-se a meta anual de visitação e definida a meta individual de 3 visitas/dia, a equipe foi dimensionada em três colaboradores, sendo um Coordenador e dois Agentes de Pós-Vendas. Para um melhor entendimento sobre o dimensionamento da equipe, segue a Tabela 2.

Tabela 2: Dimensionamento da equipe de pós-vendas

\begin{tabular}{ccccc}
$\begin{array}{l}\text { Meta } \\
\text { (visitas/ano) }\end{array}$ & $\begin{array}{l}\text { Dias Úteis } \\
\text { (ano) }\end{array}$ & $\begin{array}{c}\text { Meta Individual } \\
\text { (visitas/dia) }\end{array}$ & $\begin{array}{c}\text { Meta Individual } \\
\text { (visitas/ano) }\end{array}$ & $\begin{array}{c}\text { Dimensionamento } \\
\text { da Equipe }\end{array}$ \\
\hline 1.776 & 210 & 3 & 630 & 3 \\
\hline
\end{tabular}

Fonte: Elaborada pelo autor.

Por outro lado, o perfil das pessoas selecionadas para a equipe de pós-vendas deve apresentar algumas habilidades, as quais podem indicar uma maior adequação ao desempenho das atividades. Portanto, foram identificadas as seguintes habilidades:

a) Interpessoais: conhecer e administrar as próprias limitações; entender necessidades e desejos da outra parte; ter postura e atitude geradoras de confiança; saber comunicar-se; e estimular um clima de cooperação entre as partes envolvidas em determinado relacionamento;

b) Negociais: identificar e analisar informações; planejar, executar e controlar a visitação a clientes; ser flexível na condução de situações conflitantes; e saber 
superar impasses;

c) Técnicas: conhecer informações sobre o mercado; dominar, tecnicamente, os serviços prestados pelo Plano de Saúde; indicar soluções ou informações que facilitem a utilização dos serviços disponíveis; e entender o que é bom ou mau negócio para a empresa e para o cliente.

\subsection{A inserção do Departamento de Pós- Vendas na estrutura organizacional}

O Departamento de Pós-Vendas é subordinado à Área Comercial, juntamente com o Departamento de Vendas (internas e externas), pela qual responde o Gerente Comercial, conforme mostra a Figura 1.

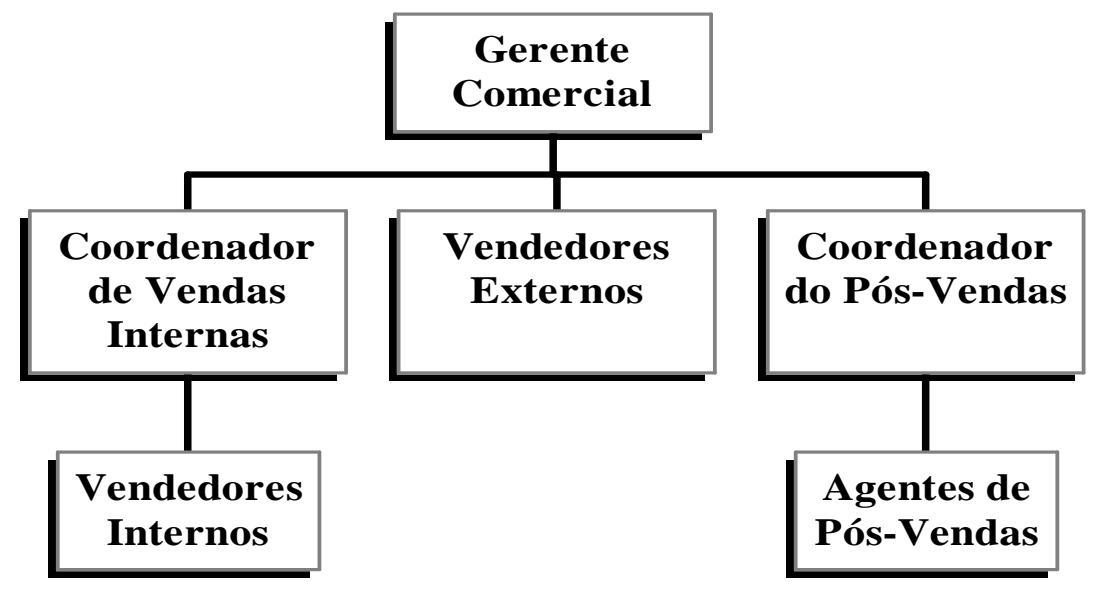

Figura 1: Organograma da Área Comercial Fonte: Elaborada pelo autor.

\subsection{Instrumentos de controle e indica- dores de desempenho}

Controlar as atividades e quantificar o desempenho operacional é imprescindível para o acompanhamento dos resultados (HRONEC, 1994; KAPLAN; NORTON, 1997; 2004). "O que não é medido não é gerenciado" (KAPLAN; NORTON, 1997, p. 21). Um nível de gestão adequado pressupõe controle e indicadores de desempenho, inclusive estes podendo ser chamados de "sinais vitais" da organização (HRONEC, 1994), fazendo-se uma alusão muito própria à área da saúde.

Com o intuito de possibilitar um controle efetivo e a análise de desempenho das atividades provenientes do Departamento de Pós-Vendas, foram definidos os seguintes instrumentos de controle e indicadores de desempenho a serem monitorados: a) Instrumentos de controle (relatórios): relatório diário das visitas; relatório descritivo semanal das visitas (por cliente); controle mensal do desempenho individual e da equipe; relatório mensal de reclamações, elogios ou sugestões; relatório mensal e acumulado da deserção de clientes; relatório das solicitações de propostas encaminhadas para o Departamento de Vendas; e

b) Indicadores de desempenho: média individual de visitas realizadas versus meta individual estipulada; total de visitas realizadas versus meta total da equipe; número de reclamações mensal e acumulado, por área ou serviço; número mensal e acumulado de solicitações de propostas encaminhadas para o Departamento de Vendas, por 
A implementação de um departamento de pós-vendas em um provedor de serviços de saúde e o estreitamento dos relacionamentos com os clientes

tipo de serviço; número e percentual de vendas concretizadas, por tipo de serviço; e número mensal e acumulado de exclusões de clientes por motivos de insatisfação versus número total de exclusões de clientes.

\subsection{Avaliações periódicas e correções no projeto}

Para atingir os objetivos propostos, foi observado um programa de avaliações sistemáticas para embasar eventuais correções no projeto. Neste sentido, a fim de verificar o desempenho e o desenvolvimento do Departamento de Pós-Vendas em suas diversas etapas, duas dimensões avaliativas se fizeram justificadas: uma voltada à avaliação do projeto como um todo, em nível diretivo, e outra à operacionalização das atividades, em nível gerencial.

A partir destas avaliações foram corrigidas ações que não convergiam com os rumos do projeto, bem como ampliando o seu escopo na medida em que a estrutura demonstrava suas potencialidades. Assim, tais dimensões de avaliação foram implementadas como segue:

a) Com a Direção: Objetivo: readequar objetivos, estrutura e re- cursos para o Departamento de Pós-Vendas, bem como seus desdobramentos estratégicos e táticos para a Área Comercial. Participantes: Diretor Executivo e Gerente Comercial. Periodicidade: inicialmente mensal e, posteriormente, semestral; e

b) Com a equipe de pós-vendas: Objetivo: prevenir e corrigir problemas operacionais, e detectar oportunidades e ameaças mercadológicas. Participantes: Gerente Comercial e equipe de pós-vendas. Periodicidade: inicialmente quinzenal e, posteriormente, mensal.

\section{Resultados e Análises}

Para análise e discussão a respeito da implementação do Departamento de Pós-Vendas são resgatados, na sequiência, os principais resultados provenientes das atividades desenvolvidas.

\subsection{Desempenho do Departamento de Pós-Vendas}

Para que se possa verificar o desempenho do Departamento de PósVendas, da equipe e individualmente, em relação à meta de visitação préestabelecida, segue a Tabela 3.

Tabela 3: Desempenho do Departamento de Pós-Vendas

\begin{tabular}{lcccccc}
\hline \multirow{2}{*}{$\begin{array}{l}\text { Equipe } \\
\text { Visitas Ano }\end{array}$} & Meta Ano 1 & (\%) Ano 1 & Visitas Ano & Meta Ano 2 & (\%) Ano 2 \\
& 1 & & \multicolumn{2}{c}{2} & \\
\hline Coordenador & 573 & 592 & 96,79 & 623 & 660 & 94,39 \\
Agente 1 & 544 & 592 & 91,89 & 683 & 660 & 103,48 \\
Agente 2 & 655 & 592 & 110,64 & 678 & 660 & 102,73 \\
\multicolumn{1}{c}{ TOTAL } & 1.772 & 1.776 & 99,77 & 1.984 & 1.980 & 100,20 \\
\hline
\end{tabular}

Fonte: Relatórios de Desempenho do Departamento de Pós-Vendas.

Conforme se observa, no ano 1 , o desempenho da equipe (1.772 visitas) em relação à meta anual (1.776) atingiu $99,77 \%$ do estabelecido, enquanto que no ano 2, o desempenho da equipe (1.984) em contrapartida à meta (1.980) alcançou $100,20 \%$ do projetado, superando a meta. Quanto ao desempenho individual, nota-se que há uma variação entre os integrantes da equipe, a qual é resultante, basicamente, 
das atribuições administrativas que sobrecarregam o Coordenador da equipe, bem como as características de perfil e qualificação das pessoas.

Além disso, verificou-se que alguns fatores interferiram significativamente no desempenho da equipe, sendo eles: adaptação do pessoal à empresa e às atividades; necessidade de ajustes nos instrumentos e processo de planejamento e controle das visitas; dificuldade no agendamento das visitas com clientes; tempo necessário para o levantamento das informações sobre os clientes, planejamento e registros das visitas; e, principalmente, entendimento inicial e apoio das gerências, chefias e colegas de trabalho no que se refere à rapidez na solução de problemas ou feedback aos clientes através da equipe de pós-vendas.

\subsection{Levantamento de reclamações}

Com a efetivação da equipe de pósvendas, o Setor de Assistência Social, até aquele momento, era o responsável pelo recebimento das reclamações, embora de forma reativa. Somente a partir do início das atividades da equipe de pós-vendas, iniciaram-se os registros das reclamações de clientes, por tipo de plano.

Dado o caráter reativo da Assistência Social em relação ao recebimento e registro das reclamações, pode-se inferir que muitos casos de insatisfação por parte dos clientes acabaram não sendo registrados nos instrumentos de controle. Sob a responsabilidade da equipe de pós-vendas, as reclamações provenientes tanto das visitas quanto aquelas encaminhadas por outras áreas da empresa, passaram a ser registradas e tratadas sistematicamente.

De acordo com a Tabela 4, as reclamações que apresentam maior freqüência são, respectivamente: cobrança de taxas de participação $(15,65 \%)$, atendimento médico $(13,64 \%)$, cadastro $(10,27 \%)$, Pronto Atendimento $(9,76 \%)$ e número de médicos $(9,10 \%)$; o que representa $58,42 \%$ do total das reclamações

Tabela 4: Registro de reclamações a partir da implementação da equipe de pós-vendas

\begin{tabular}{lcccccc}
\hline Tipo de Reclamação & Ano 1 & \% & Ano 2 & & Total & \% Geral \\
\hline Cobrança de taxas de participação & 38 & 10,30 & 55 & 24,45 & 93 & 15,65 \\
Atendimento médico & 69 & 18,70 & 12 & 5,33 & 81 & 13,64 \\
Cadastro & 40 & 10,84 & 21 & 9,33 & 61 & 10,27 \\
Pronto Atendimento & 35 & 9,49 & 23 & 10,22 & 58 & 9,76 \\
Número de médicos & 25 & 6,77 & 29 & 12,89 & 54 & 9,10 \\
Demais reclamações & 162 & 43,90 & 85 & 37,78 & 247 & 41,58 \\
$\quad$ TOTAL & 369 & 100 & 225 & 100 & 594 & 100
\end{tabular}

Fonte: Relatórios de Reclamações.

Com o conhecimento do tipo e da freqüência das reclamações, medidas corretivas foram adotadas, fato este que explica a queda considerável no número absoluto das reclamações ao se comparar os anos 1 e 2 . Analisando o levantamento das reclamações, percebe-se que a média mensal de reclamações do ano $2(18,75)$ diminuiu em relação ao ano $1(30,75)$, havendo, portanto, uma queda de 39,02\% sobre o total de reclamações.

Outro aspecto que se evidencia na Tabela 4 é a redução nos indicadores de reclamações de clientes quanto aos 
A implementação de um departamento de pós-vendas em um provedor de serviços de saúde e o estreitamento dos relacionamentos com os clientes

atributos do serviço: atendimento médico, cadastro e Pronto Atendimento, traduzindo, na percepção do cliente, de certa forma, a melhoria no nível de qualidade dos serviços com base em alterações implementadas no processo de prestação de serviços.

\subsection{Encaminhamentos de solicitações de propostas para o Departamento de}

\section{Vendas}

Além de fortalecer o relacionamento com os clientes, a equipe de pósvendas exerce um papel fundamental no que diz respeito ao incremento de receitas da empresa. Conforme mostra a Tabela 5, pode-se verificar o número de encaminhamentos de solicitações de propostas para o Departamento de Vendas, bem como o fechamento de novos negócios.

Tabela 5: Propostas encaminhadas e fechamento de novos negócios

\begin{tabular}{lccccc}
\hline Tipos de Serviço & $\begin{array}{c}\text { Propostas } \\
\text { Ano 1 }\end{array}$ & $\begin{array}{c}\text { Propostas } \\
\text { Ano 2 }\end{array}$ & $\begin{array}{c}\text { Total de } \\
\text { Propostas }\end{array}$ & $\begin{array}{c}\text { Total de } \\
\text { Vendas }\end{array}$ & $\begin{array}{c}\text { Fechamento } \\
\text { Fenam }\end{array}$ \\
\hline Plano de Saúde & 36 & 19 & 55 & 27 & 49,09 \\
Medicina do Trabalho & 22 & 32 & 54 & 33 & 61,11 \\
Acidente do Trabalho & 24 & 29 & 53 & 11 & 20,75 \\
Odontologia & 29 & 8 & 37 & 9 & 24,32 \\
Farmácia & 35 & 22 & 57 & 40 & 70,18 \\
& 146 & 110 & 256 & 120 & 46,88 \\
\hline
\end{tabular}

Fonte: Relatórios de Solicitações de Propostas para o Departamento Vendas.

Ao longo desses dois anos, foram encaminhadas 256 solicitações de propostas pela equipe de pós-vendas para o Departamento de Vendas. Deste total, 120 propostas foram efetivamente concretizadas, perfazendo um indicador de 46,88\% de sucesso no fechamento das propostas encaminhadas. Cabe ressaltar que, as propostas encaminhadas são relativas a solicitações de serviços ofertados pela empresa que os clientes atuais, ao serem contatados pela equipe de pós-vendas, ainda não possuíam.

\subsection{Números e motivos que causam a deserção de clientes}

Conhecer os motivos causadores da deserção de clientes é condição básica para a tomada de decisões que consolide a satisfação e a retenção de clientes. Sendo assim, a partir do ano 1, de acordo com Tabela 6, foram levantados a quantidade e os motivos da deserção (ou perda) de clientes.

Tabela 6: Motivos da deserção de clientes

\begin{tabular}{lcccccc}
\hline & Ano 1 & & & & \\
Motivos da Deserção (ou Perda) de Clientes & & $\%$ & Ano 2 & $\%$ & Total & $\%$ \\
\hline Abrangência geográfica (cobertura dos serviços) & 4 & 5,19 & 1 & 1,75 & 5 & 3,73 \\
Atendimento & 3 & 3,90 & 4 & 7,02 & 7 & 5,22 \\
Concorrência & 23 & 29,87 & 5 & 8,77 & 28 & 20,90 \\
Inadimplência & 24 & 31,17 & 20 & 35,09 & 44 & 32,83 \\
Redução de gastos & 11 & 14,29 & 5 & 8,77 & 16 & 11,94 \\
Desconhecido & 6 & 7,79 & 0 & 0 & 6 & 4,48 \\
Outros & 6 & 7,79 & 22 & 38,60 & 28 & 20,90
\end{tabular}


Com a atuação da equipe de pósvendas, além do registro quantitativo sobre a deserção de clientes, passou-se a verificar quais eram os motivos da solicitação de cancelamento do contrato por parte dos clientes. $\mathrm{Na}$ maioria dos casos, os clientes explicaram os motivos de sua decisão, havendo situações em que a equipe de pós-vendas conseguiu revertê-la.

Tendo em vista que os motivos "inadimplência" $(32,83 \%)$ e "redução de gastos" $(11,94 \%)$, respondendo por $44,77 \%$ dos cancelamentos contratuais, podem ser entendidos como de difícil reversão ou de reversão indesejada, restam $55,23 \%$ dos casos que devem ter seus motivos trabalhados pela empresa, dentre os quais o mais representativo é a concorrência (20,90\%). Entretanto, paralelamente à diminuição nas reclamações do ano 2 em relação ao ano 1, o mesmo ocorreu em termos de deserção (perda) de clientes, sinalizando uma queda de $25,97 \%$.

\subsection{As entrevistas com clientes}

\subsubsection{Caracterização dos entrevistados}

As entrevistas individuais em profundidade desenvolvidas com clientes da operadora de planos de saúde foi direcionada ao segmento Plano de Saúde Empresarial. Sendo assim, foram selecionados, aleatoriamento, três clientesempresa. Tais clientes, e os respectivos entrevistados, apresentam as seguintes características:

a) Entrevistado A: o entrevistado é o Gerente de Recursos Humanos de uma empresa do setor moveleiro, exercendo o papel de negociador nos processos de contratação de benefícios para os seus colaboradores. A empresa foi fundada em 1983, e atua nos segmentos de móveis e divisórias para escritório. Além do mercado nacional, exporta seus produtos para três países do Mercosul: Argentina, Paraguai e Uruguai;

b) Entrevistado B: o entrevistado é um dos proprietários de uma empresa do ramo gráfico, exercendo o papel, tanto de decisor, quanto de negociador nos processos de contratação de benefícios para os seus colaboradores. A empresa foi fundada em 1990 e dá ênfase aos segmentos de propaganda e editorial, sendo que o seu mercado é compreendido pela região da Serra Gaúcha e Grande Porto Alegre (RS);

c) Entrevistada C: a entrevistada é um dos proprietários de uma empresa do comércio atacadista de bebidas, assumindo a função, tanto de decisão, quanto de negociação nos processos de contratação de benefícios para os seus colaboradores. A empresa foi fundada em 1997 e possui três linhas de produto: refrigerante, cerveja e água mineral. A abrangência territorial do seu mercado é compreendida pelas principais cidades da Serra Gaúcha. Seus principais fornecedores são: AMBEV e PepsiCola.

\subsubsection{A Percepção de alguns clientes quanto à atuação da equipe de pós- vendas}

Em relação aos contatos com a equipe de pós-vendas, o Entrevistado A afirmou estar recebendo visitas sistemáticas e sempre que precisou de informações ou a resolução de algum 
A implementação de um departamento de pós-vendas em um provedor de serviços de saúde e o estreitamento dos relacionamentos com os clientes

problema, sempre fora bem atendido. Quanto ao tempo de retorno às suas solicitações, na maioria dos casos, foi satisfatório.

[...] o contato com a equipe de Pós-Vendas é tranqüilo, talvez até em função da pessoa que nos visita, pois já a conhecemos há bastante tempo, e a relação é bem próxima.

Como benefícios percebidos através das atividades desenvolvidas pela equipe de Pós-Vendas, foram identificados: agilidade nas informações, viabilizando soluções mais rápidas para os problemas; e centralização na comunicação, argumentando que:

[...] saber com quem falar e solicitar alguma providência abrevia o tempo de retorno.

Argüido sobre qual a sua opinião quanto à equipe de pós-vendas ser um investimento de longo prazo, por parte do Plano de Saúde, no relacionamento com sua empresa, o entrevistado disse que entende que sim, e salientou que:

[...] sem dúvida, entendo que não é somente um investimento, mas também um diferencial do Plano em relação a outros prestadores de serviço que a empresa tem como parceiros. Acredito que a equipe de Pós-Vendas reforça o relacionamento entre as empresas, bem como a imagem do Plano.

Para o entrevistado, tanto a confiança, quanto o compromisso em permanecer com o atual convênio foram consolidados, uma vez que as atividades desenvolvidas pela equipe de pós-vendas agregam valor.

Na realidade já existia uma con- fiança muito grande, mas a equipe de Pós-Vendas a fortaleceu, o mesmo se aplicando ao compromisso. Nós sentimos a preocupação do Plano em manter um relacionamento, e o pósvendas veio somar esforços, embora ainda estarmos utilizando-o mais para resolver problemas. Temos a impressão de que o Plano está profissionalizando sua estrutura, cada vez mais, melhorando os processos.

O entrevistado afirmou, também, haver uma forte propensão da empresa em permanecer com o atual convênio, em uma perspectiva de futuro.

Dentro de um contexto geral, não somente pela atuação do Pós-Vendas, existe uma forte propensão em permanecermos como clientes do Plano de Saúde. Se estivermos bem atendidos e as pessoas satisfeitas, não há o porquê de pensarmos em trocar de parceiro.

Questionado sobre os contatos com a equipe de Pós-Vendas, o Entrevistado B comentou que recebera, até o momento, quatro visitas, e sempre que precisou de informações ou a resolução de algum problema, sempre fora bem atendido, colocando ainda que o tempo de retorno às solicitações foi satisfatório.

O contato com a pessoa de PósVendas personalizou o atendimento. Conhecer as pessoas do Plano facilita o atendimento e diminui a distância entre as pessoas e o Plano, criando um vínculo.

Como benefícios percebidos através das atividades desenvolvidas pela equipe de pós-vendas, foram identificados: aproximação da empresa com o Plano, 
sendo esse ressaltado como o principal benefício, e resgate das informações sobre coberturas, direitos e procedimentos para a melhor utilização dos serviços, melhorando a comunicação. Em relação à percepção da equipe de pós-vendas como um investimento de longo prazo por parte do Plano de Saúde, no relacionamento com sua empresa, o entrevistado entende que sim.

[...] o pós-vendas está aproximando as empresas. Como os concorrentes têm interesse, anterior às visitas realizadas pela equipe, recebíamos mais visitas de vendedores dos concorrentes do que do Plano de Saúde. Este contato é um ponto positivo e entendo que este tipo de postura reforçará, cada vez mais, o vínculo entre as empresas.

Em acréscimo, o entrevistado declinou que sua percepção, tanto de confiança, quanto de compromisso em permanecer com o atual convênio, aumentaram a partir da aproximação da equipe de pósvendas. Neste sentido, o entrevistado comentou que:

Percebe-se um grande interesse do Plano em permanecer com a empresa como cliente, uma coisa que não vinha sendo demonstrada, anteriormente, e isto é fundamental. Acredito que há um investimento de longo prazo de ambas as partes, pois um convênio com plano de saúde não deve ser trocado de um mês para outro. A visão que o Plano está nos transmitindo é que o relacionamento é uma continuação de uma parceria, por muito tempo.

Finalmente, indagado sobre a tendência de sua empresa em permanecer com o atual convênio de Plano de Saúde, no longo prazo, afirmou estar surpreso pela atenção que vem recebendo, uma vez que após nove anos como cliente nunca havia sido visitado, e que tal aproximação e sua valorização como cliente, aliada à qualidade do atendimento prestado, reforça sua intenção em manter o convênio.

A Entrevistada C, por sua vez, questionada a respeito dos contatos com a equipe de pós-vendas, disse ter recebido três visitas e sempre que precisou dirimir alguma dúvida, enfatizando as taxas de participação, foi prontamente atendida, ressaltando que, não apenas pela equipe de pós-vendas, mas também pelo Setor de Assistência Social.

Como benefícios percebidos através das atividades desenvolvidas pela equipe de pós-vendas, foram identificados: aproximação da empresa com o Plano e, principalmente, disponibilidade de um canal de contato, no qual o cliente possa ser ouvido. Com base nestes benefícios, a entrevistada comentou que:

[...] a equipe de pós-vendas possibilita que o cliente conheça melhor os seus direitos, como proceder em alguns casos específicos e com quem deve tratar, proporcionando contatos mais direcionados.

Ao ser indagada quanto à sua percepção sobre a equipe de pós-vendas ser um investimento de longo prazo, por parte do Plano de Saúde, no relacionamento com sua empresa, a entrevistada disse entender que sim, colocando que:

[...] como toda empresa depende muito de seus clientes e todos gostam de ser ouvidos e valorizados, a equipe de pósvendas torna-se um vínculo entre o Plano e a empresa. A atenção e o retorno às solicitações provenientes da atuação do pósvendas são essenciais. Saber a quem recorrer, tendo a seguran- 
A implementação de um departamento de pós-vendas em um provedor de serviços de saúde e o estreitamento dos relacionamentos com os clientes

ça de que algum problema que ocorra vai ser resolvido faz com que a nossa tolerância a alguns erros aumente.

Além disso, a entrevistada comentou que sua percepção, tanto de confiança, quanto de compromisso em permanecer com o atual convênio, não mudaram, devido ao fato de considerar que ambas apresentam um nível alto, tendo em vista a sua proximidade com o Plano de Saúde, reforçando que "sente-se em casa". Contudo, salientou que a equipe de pós-vendas, embora não tenha alterado sua percepção de confiança e compromisso, certamente fortalece o relacionamento entre as empresas. Concluindo, ao ser questionada sobre a tendência de sua empresa em permanecer com o atual convênio de Plano de Saúde, no longo prazo, afirmou haver uma forte tendência em manter o convênio.

\section{Considerações finais}

Observando uma evolução constante na condução dos trabalhos desenvolvidos pela equipe de pós-vendas, bem como a necessidade de outros elementos serem incorporados à sua atuação, como, por exemplo, expandir as atividades para associados do Plano de Saúde Individual e estruturar um indicador para a aferição da retenção de clientes, nota-se que o objetivo central proposto pelo projeto, o de estabelecer contatos com clientes, fortalecendo um relacionamento de longo prazo que vise a sua retenção, está sendo, gradativamente, alcançado.

É imperioso ressaltar que a participação efetiva da equipe de pós-vendas como meio de estimular melhorias em processos e na qualidade dos serviços ofertados, resgatando a percepção dos clientes, vem se consolidando, o que é fundamentado pelos indicadores relativos a reclamações e motivos causadores da deserção de clientes, os quais vêm obtendo registros decrescentes; bem como para o incremento da receita da empresa, o que se observa pelo número de solicitações de propostas encaminhadas pela equipe de pós-vendas para o Departamento de Vendas.

Pelas entrevistas com alguns clientes da empresa, observa-se que, quanto à influência da prática do Departamento de Pós-Vendas sobre a retenção de clientes, ou melhor, sobre o desejo futuro dos clientes permanecerem engajados com o atual provedor de saúde, todos os entrevistados afirmaram haver forte tendência na sua permanência como clientes do Plano de Saúde. Embora as ações advindas da equipe de pós-vendas não sejam responsáveis, exclusivamente, pela retenção de clientes, os mesmos declinaram que tais iniciativas são bastante valorizadas.

Vale salientar que, para a prática de pós-vendas desenvolvida pela empresa em estudo, a retenção de clientes não é um fim em si mesma, mas o resultado desejado ao final da consolidação de relacionamentos com clientes sob uma perspectiva relacional de longo prazo. Por isso, as atividades de pós-vendas devem focar, em essência, os relacionamentos, tendo como resultados pretendidos ou conseqüências de relacionamentos estreitos e sistemáticos com os clientes, então, a satisfação e, principalmente, a retenção dos clientes.

Nesse horizonte, Rowe e Barnes (1998) propõem que um relacionamento construído sobre os conceitos da mutualidade (benefícios mútuos) e do status especial (atendimento diferenciado e/ou personalizado) tem potencial para gerar uma vantagem competitiva sustentável para a empresa. Sendo assim, a comunicação com os clientes ajuda no desenvolvimento do relacionamento, fomentando a confiança e propiciando informações e conhecimento necessários aos parceiros para o empreendimento de atividades cooperativas e colaborativas (PARVATIYAR; SHETH, 2000; JIANG; HENNEBERG; NAUDÉ, 2011; TAI, 2011). Por isso, ressalta-se a importância dos clientes terem percebido como um dos benefícios da disponibilização da equipe de pós-vendas a melhoria no nível 
de comunicação e das informações prestadas pelo provedor de serviços.

Por se tratar de um estudo exploratório, é oportuno comentar que os achados de pesquisa não podem ser generalizados, mas sim, servir apenas como evidências pontuais de uma situação na qual a prática de estreitamento e consolidação de relacionamentos entre uma empresa e seus principais clientes vem ocorrendo de forma positiva. Como limitações do estudo, portanto, é possível citar, por exemplo, o pequeno número de clientes entrevistados e o volume de dados coletados, por meio da análise documental, que cobriu somente o horizonte de dois anos a partir da implementação do departamento de pós-vendas da empresa.

No sentido de se buscar evidências mais robustas, cabe registrar a possibilidade do desenvolvimento de futuras pesquisas. Uma delas seria a aplicação de uma abordagem quantitativo-descritiva, por meio de uma survey, preferencialmente de forma longitudinal, para se verificar o grau de satisfação dos clientes em relação aos serviços de pós-vendas disponibilizados pela empresa. Outras possibilidades a serem destacadas são pesquisas sobre a imagem e a reputação da empresa a partir da introdução formal dos serviços de pósvendas em sua estrutura; e estudos que aprofundassem o entendimento de construtos tais como valor percebido, comprometimento, confiança, retenção e lealdade de clientes a partir da agregação de valor no atendimento aos clientes com base na atuação da equipe de pós-vendas. Também seriam relevantes estudos que contemplassem construtos de impacto negativo no contexto da prática do marketing de relacionamento. Um exemplo disso seria o de analisar se pela maior proximidade da empresa junto aos seus clientes, a partir da atuação da equipe de pós-vendas, a incidência de comportamentos oportunistas e atos destrutivos diminuiu entre as partes.

\section{Referências}

BAHIA, Ligia; LUIZ, Ronir Raggio. Informações sobre coberturas e preços de planos e seguros privados de saúde: as perspectivas da complementaridade entre fontes administrativas e de base populacional. Ciência \& Saúde Coletiva, v. 11, n. 4, p. 878-880, 2006.

BERRY, Leonard L. Relationship marketing of services - perspectives from 1983 and 2000. Journal of Relationship Marketing, v. 1, n. 1, p. 59-77, 2002.

BISOGNANO, Maureen. New skills needed in medical leadership. Quality Progress, v. 33, n. 6, p. 32-41, 2000.

CASANOVA, Robert. Patient satisfaction: designing and managing your patients' experience. Annual Quality Congress, 53., ASQC, Anaheim, p. 386-388, 1999.

CHAHAL, Hardeep; KUMARI, Neetu. Consumer perceived value and consumer loyalty in the healthcare sector. Journal of Relationship Marketing, v. 10, n. 2, p. 88-112, 2011.

CLINEFELTER, Kathryn. Future perspectives for healthcare quality professionals. Annual Quality Congress, 54., ASQC, Indianapolis, p. 804-806, 2000.

COELHO, Pedro Simões; HENSELER, Joerg. Creating customer loyalty through service customization. European Journal of Marketing, v. 46, n. 3/4, p. 331-356, 2012.

COOPER, Donald R.; SCHINDLER, Pamela S. Métodos de pesquisa em administração. 10. ed. Porto Alegre: Bookman, 2011.

DAY, George S. Managing market relationships. Journal of the Academy of Marketing Science, v. 28, n. 1, p. 24-30, 2000 .

DWYER, F. Robert; SCHURR, Paul H.; $\mathrm{OH}$, Sejo. Developing buyer-seller rela- 
A implementação de um departamento de pós-vendas em um provedor de serviços de saúde e o estreitamento dos relacionamentos com os clientes

tionships. Journal of Marketing, v. 51, n. 2, p. 11-27, 1987.

EDWARD, Manoj; SHADEV, Sunil. Role of switching costs in the service quality, perceived, value, customer satisfaction and customer retention linkage. Asia Pacific Journal of Marketing and Logistics, v. 22, n. 3, p. 327-345, 2011.

EISENBERG, Barry. Customer service in healthcare: a new era. Hospital \& Health Services Administration, v. 42, n. 1, p. 17-31, 1997.

FOLLAND, Shermann; GOODMAN, Allen C.; STANO, Miron. A economia da saúde. 5. ed. Porto Alegre: Bookman, 2008.

GAUR, Sanjaya Singh; XU, Yingzi; QUAZI, Ali; NANDI, Swathi. Relational impact of service providers' interaction behavior in healthcare. Managing Service Quality, v. 21, n. 1, p. 67-87, 2011.

GREGÓRIO, Renato. Marketing médico: criando valor para o paciente. Rio de Janeiro: Editora DOC, 2009.

GORDON, Ian. Customer relationship management: conflict to collaboration. Ivey Business Journal, v. 65, n. 5, p. 911, 2001.

GRÖNROOS, Christian. Relationship marketing: the nordic school perspective. In: SHETH, Jagdish N.; PARVATIYAR, Atul (eds.). Handbook of relationship marketing. Thousand Oaks: Sage Publications, 2000. cap. 4, p. 95-117.

GUMMESSON, Evert. Implementation requires a relationship marketing paradigm. Journal of the Academy of Marketing Science, v. 26, n. 5, p. 242-249, 1998.

GUMMESSON, Evert. Are current research approaches in marketing leading us astray? Marketing Theory, v. 1, n. 1, p. 27-48, 2001.

HAIR Jr., Joseph F.; WOLFINBARGER, Mary; ORTINAU, David J.; BUSH, Robert P. Fundamentos de pesquisa de marketing. Porto Alegre: Bookman, 2010.
HENNINK, Monique; HUTTER, Inge; BAILEY, Ajay. Qualitative research methods. Thousand Oaks: Sage Publications, 2011.

HESKETT, James L.; JONES, T. O.; LOVEMAN, G. W.; SASSER Jr., W. Earl; SCHLESINGER, Leonard A. Putting the service-profit chain to work. Harvard Business Review - Business Classics: Fifteen Key Concepts for Managerial Success, p.110-120, 1994.

JIANG, Zhizhong; HENNEBERG, Stephan C.; NAUDÉ, Peter. The importance of trust vis-à-vis reliance in business relationships: some international findings. International Marketing Review, v. 28, n. 4, p. 318-339, 2011.

HRONEC, Steven M. Sinais vitais: usando medidas do desempenho da qualidade, tempo e custo para traçar a rota para o futuro de sua empresa. São Paulo: Makron Books, 1994.

KAPLAN, Robert S.; NORTON, David P. A estratégia em ação: balanced scorecard. 4. ed. Rio de Janeiro: Campus, 1997.

KAPLAN, Robert S.; NORTON, David P. Mapas estratégicos: convertendo ativos intangíveis em resultados tangíveis. 2. ed. Rio de Janeiro: Elsevier, 2004.

KERNS, Charles D.; ST. CLAIR, Rod. Loyalty in managed care: a leadership system. Journal of Healthcare Management, v. 45, n. 3, p. 158-168, 2000.

KING, Nigel; HORROCKS, Christine. Interviews in qualitative research. Thousand Oaks: Sage Publications, 2010.

KOTHANDARAMAN, P.; WILSON, D. $\mathrm{T}$. Implementing relati-onship strategy. Industrial Marketing Management, v. 29, n. 4, p. 339-349, 2000.

KOTLER, Philip; SHALOWITZ, Joel; STEVENS, Robert J. Marketing estratégico para a área da saúde: a construção de um sistema voltado ao cliente. Porto Alegre: Bookman, 2010.

LAM, Shun Yin; SHANKAR, Venkatesh; 
ERRAMILLI, M. Krishnna; MURTHY, Bvsan. Customer value, satisfaction, loyalty, and switching costs: an illustration from a business-to-business service context. Journal of the Academy of Marketing Science, v. 32, n. 3, p. 293-311, 2004.

LIN, Jiun-Sheng; WU, Chung-Yueh. The role of expected future use in relationshipbased service retention. Managing Service Quality, v. 21, n. 5, p. 535-551, 2011.

MALLOCK, Kathy; LUSSIER, James T. Healing models for organizations: description, measurement, and outcomes. Journal of Healthcare Management, v. 45, n. 5, p. 332-346, 2000.

MEYER, J. Using qualitative methods in health related action research. British Medical Journal, v. 320, n. 7.228, p. 178$182,2000$.

MILAN, Gabriel Sperandio. A retenção de clientes como resultado da prática do marketing de relacionamento. In: MILAN, Gabriel Sperandio; BRANCHI, Nelson Vinicius Lopes (org.). Administração mercadológica: teoria e pesquisas. Caxias do Sul, EDUCS, 2004. cap. 8, 177-195.

MILAN, Gabriel Sperandio; DE TONI, Deonir. A construção de um modelo sobre a retenção de clientes e seus antecedentes em um ambiente de serviços. REAd Revista Eletrônica da Administração, v. 18, n. 2, p. 433-467, 2012.

MILAN, Gabriel Sperandio; RIBEIRO, José Luis Duarte. A influência do departamento de pós-vendas na retenção de clientes: um estudo qualitativo em uma empresa de medicina de grupo. Gestão \& Produção, v. 10, n. 2, p. 197-216, 2003.

MILAN, Gabriel Sperandio; TREZ, Guilherme. Pesquisa de satisfação: um modelo para planos de saúde. RAEeletrônica, v. 4, n. 2, p. 1-21, 2005.

MILAN, Gabriel Sperandio. A prática do marketing de relacionamento e a retenção de clientes: um estudo aplicado em um ambiente de serviços. Tese de Doutorado. Porto Alegre: Universidade Federal do Rio Grande do Sul, 2006.

MORGAN, Robert M.; HUNT, Shelby D. The commitment-trust theory of relationship marketing. Journal of Marketing, v. 58, n. 3, p. 20-38, 1994.

MORRISON, B. How can action research apply to health services? Qualitative Health Research, v. 11, n. 4, p. 436-450, 2001.

OLIVER, Richard L. Satisfaction: a behavioral perspective on the consumer. New York: M. E. Sharpe, 2010.

PARVATIYAR, Atul; SHETH, Jagdish N. The domain and conceptual foundations of relationship marketing. In: SHETH, Jagdish N.; PARVATIYAR, Atul (eds.). Handbook of relationship marketing. Thousand Oaks: Sage Publications, 2000. cap. 1, p.3-38.

PIVA, Luciana Cláudia; FUMAGALLI, Luis André Wernecke; BAPTISTA, Paulo De Paula; SILVA, Wesley Vieira. Relação entre satisfação, retenção e rentabilidade de clientes no setor de planos de saúde. RCA - Revista de Ciências da Administração, v. 9, n. 19, p. 54-80, 2007.

REICHHELD, Frederick F. The loyalty effect: the hidden force behind growth, profits, and lasting value. Boston: Harvard Business School Press, 1996a.

REICHHELD, Frederick F. Learning from customer defections. Harvard Business Review, v. 74, n. 2, p. 56-69, 1996 b.

REICHHELD, Frederick F.; SASSER Jr., Earl W. Zero defections: quality comes to service. Harvard Business Review, v. 68, n. 5, p. 105-111, 1990.

REICHHELD, Frederick F.; MARKEY Jr., Robert G.; HOPTON, Christopher. The loyalty effect: the relationships between loyalty and profits. European Business 
A implementação de um departamento de pós-vendas em um provedor de serviços de saúde e o estreitamento dos relacionamentos com os clientes

Journal, v. 12, n. 3, p. 134-139, 2000.

RIBEIRO, José Luis Duarte; MILAN, Gabriel Sperandio. Planejando e conduzindo entrevistas individuais. In: RIBEIRO, José Luis Duarte; MILAN, Gabriel Sperandio (eds.). Entrevistas individuais: teoria e aplicações. Porto Alegre: FEEng/UFRGS, 2004.

ROWE, Glenn W.; BARNES, James G. Relationship marketing and sustained competitive advantage. Journal of Market Focused Management, v. 2, n. 3, p. 281-297, 1998.

SCOTT, Greg; GARNER, Roberta. Doing qualitative research: designs, methods, and techniques. 1. ed. Upper Saddle River: Pearson, 2013.

SPILLER, Eduardo Santiago; SENNA, Anna Maria; SANTOS, José Ferreira; VILAR, Josier Marques. Gestão dos serviços de saúde. 1. ed. Rio de Janeiro: Editora FGV, 2009.

TAI, Yi-Ming. Perceived value for customers in information sharing services. Industrial Management \& Data Systems, v. 111, n. 4, p. 551-569, 2011.

THIOLLENT, Michel. Pesquisa-ação nas organizações. 2. ed. São Paulo: Atlas, 2009.

VAVRA, Terry G. Selling after the sale: the advantages of aftermarketing. Supervision, v. 55, n. 10, p. 9, 1994.

VAVRA, Terry G.; PRUDEN, Douglas R. Using aftermarketing to maintain a customer base. Discount Merchandiser, v. 35, n. 5, p. 86, 1995.

VERGARA, Sylvia Constant. Projetos e relatórios de pesquisa em administração. 11. ed. São Paulo: Atlas, 2009.

WILLIAMS, Paul; NAUMANN, Earl. Customer satisfaction and business performance: a firm-level analysis. Journal of Services Marketing, v. 25, n. 1, p. 20-32, 2011.
WILSON, Timothy L. International aftersales services. Journal of Global Marketing, v. 13, n. 1, p. 5-27, 2008.

WOLF, Emily J. A new approach to healthcare marketing. Healthcare Executive, v. 16, n. 1, p. 12-15, 2001a.

WOLF, Emily J. Four strategies for successful recruitment and retention. Healthcare Executive, v. 16, n. 4, p. 1418, 2001b.

WOLF, Emily J. Collaborative strategies. Healthcare Executive, v. 18, n. 1, p. 1215, 2003. 